\title{
Infrared Image Stitching Based on Immune Memory Clonal Selection Algorithm
}

\author{
by Tong Hejun, Fu Dongmei, Dong Lin and Yang Tao
}

School of Automation and Electrical Engineering, University of Science and Technology Beijing, Beijing 100083, P. R. China, tonghejun@126.com

\begin{abstract}
Due to the limitations of infrared instrument sampling window size and shooting distance, collection of largescale target often need multiple filming and multiple infrared image stitching. Since infrared image blur itself, the image stitching process exists the difficulty of feature points extraction and matching points confirmation. Faced with this problem we proposed an image stitching algorithm based on immune memory clonal selection. We improved Susan algorithm to extract feature points, and got matching points by the proposed algorithm. Simulation results show that the algorithm can obtain a high rate of matching points, so as to achieve a good performance.
\end{abstract}

\section{Introduction}

Since infrared instrument wide-angle, pixels and other objective factors, the capture infrared images often do not show the full target. Thus the two or multiple infrared images stitching into one seamless image is essential [1,2]. Seamless image stitching often requires the following steps: firstly, try to select out the completely corresponding feature points (at least two, but also can be more) in the two stitching images as matching points or stitching points and this process is known as image registration. Secondly, stitch the two images in accordance with the matching points. Finally, if the stitching result exist the phenomenon of brightness uneven or gray level uneven, we need to fuse the overlap part in order to realize the seamless stitching. Obviously, image registration is the core technology of the image stitching and it is directly related with the splicing speed and the success rate.

Stitching algorithm diversity is based on the utilization of different image information in the process of image registration [3], which can be roughly divided into: (1) Method based on gray level information, such as Ratio Matching method [4], Grid Matching method[5], etc. These algorithms calculate the match points' surrounding area, so the calculation amount is large and the speed is slow; (2) Method based on transform domain method, such as De Castro and Morandi's extension phase correlation method [6], Reddy and Chattcrii proposed method based on fast Fourier transform [7], etc. The phase correlation method calculates simple and accurate, but it is required larger overlap ratio between registration images and the image size influences calculation amount and applicable scope; (3) Method based on the characteristics, such as Harris algorithm [8], Susan algorithm [9], etc. This method extracts the image feature points, search matching points from the feature points, complete image stitching. The advantage of this approach is that greatly reduces the match time, the image has good stability, have certain ability to resist disturbance on position transformation, etc.

However because of the characteristics of infrared image, the selection of feature points and matching point becomes difficult. Traditional Susan algorithm need to set up different thresholds for different images, and produce too much feature points, increase the algorithm running time and effort. Literature[10] reference the memory feature and clonal selection optimization of the immune system and put forward an improved clonal selection algorithm, but the method did not explicitly gives its affinity function in the choice of two weighting coefficient method, brought difficulties for the choice of algorithm of feature points. In this paper, on the basis of the implementation of the improved Susan algorithm, the selection of feature points is not affected by images of light and shade, and it can limit number of feature points. Immune memory clonal selection algorithm (IMCSA) is improved, so that the affinity function of the weighted coefficients can be adaptive adjustment, improve the matching accuracy and ability to resist deformation.

\section{Adaptive Susan algorithm to extract image features $[11,12,13]$}

Susan algorithm, the Smallest Univalue Segment Assimilating Nucleus, was put forward by Smith and Brady. The basic principle is to create a circular template, template center as template nuclear, calculate the gray relation between each point and template nuclear, based on the template with similar to nuclear or identical grey value of some form of similar to an area of judgment and template to determine the location of flat, edge or corner.

Susan template slide in the image, compare each image pixel brightness in the template and the brightness of the template nuclear in every position:

$$
C\left(\vec{r}, \vec{r}_{0}\right)=\left\{\begin{array}{l}
1, \text { if }\left|I(\vec{r})-I\left(\vec{r}_{0}\right)\right| \leq t \\
0, \text { if }\left|I(\vec{r})-I\left(\vec{r}_{0}\right)\right|>t
\end{array}\right.
$$


In Eq. (1), $\quad \vec{r}_{0}$ is the nuclear in 2D image; $\vec{r}$ is the other points except the nuclear in the template; $I(\vec{r})$ is the brightness of $\vec{r} ; I\left(\vec{r}_{0}\right)$ is the brightness of nuclear; $t$ is the brightness difference threshold and the choice of $t$ is closely related to the contrast of the image. In this article, we take the mean gradient of each image as the brightness difference threshold to adapt different light and shade; $c\left(\vec{r}, \vec{r}_{0}\right)$ is the result of brightness compare.

The sum of brightness compare result between all points in the template $(n)$ and the nuclear is

$$
n\left(\vec{r}_{0}\right)=\sum_{\vec{r}} c\left(\vec{r}, \vec{r}_{0}\right)
$$

Then compare $n\left(\vec{r}_{0}\right)$ with a given threshold $g$ (Called the geometric threshold), and get the image edge response:

$$
R\left(\vec{r}_{0}\right)= \begin{cases}g-n\left(\vec{r}_{0}\right), & \text { if }\left|n\left(\vec{r}_{0}\right)\right|<g \\ 0, & \text { else }\end{cases}
$$

$\vec{r}_{0}$ is the feature point. The choice of $g$ is also a problem, so this paper proposes a method based on loop iteration to automatically determine the appropriate $g$ values. The process is: first, given a default initial threshold $g$, a desired feature points $\mathrm{N}$; next, use Susan algorithm to obtain feature points, if the feature points more than $\mathrm{N}$, then update the threshold $g^{\prime}=g^{*} 0.9$; finally, use the latest threshold calculation feature points, until the feature points is less than $\mathrm{N}$, at this time of the geometric threshold as the best threshold value.

Traditional Susan algorithm need manually setting the brightness difference threshold $t$ and geometry threshold $g$, while the improved Susan algorithm proposed in this paper can set adaptive threshold according to the image. Brightness difference threshold is closely related to the brightness of the image, so we use mean gradient of each image as the threshold to adapt different shade. This paper proposes a method based on loop iteration to automatically determine the appropriate values of geometry threshold. In Figure 1, (a) (c) have the same gray values and (b) (d) have the same gray values. (a) (b) set the same threshold manually but the extracted feature points $n$ vary widely. (c) (d) set adaptive threshold and the result of feature points extraction is good.

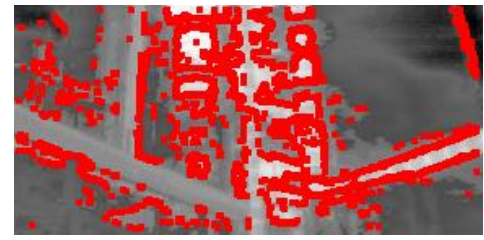

(a) $t=10, g=7.2, n=2679$

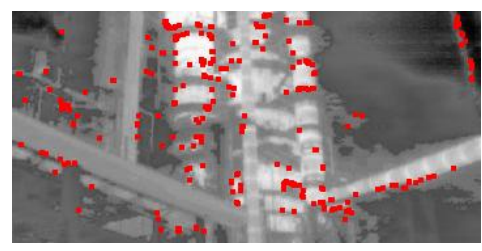

(c) $t=8.68, g=2.88, n=272$

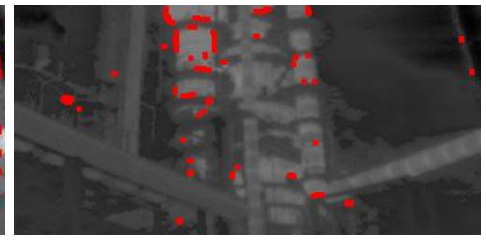

(b) $t=10, g=7.2, n=167$

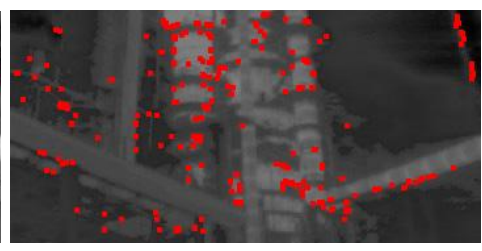

(d) $t=4.37, g=2.88, n=279$

Fig. 1. Adaptive Susan algorithm to extract image features

\section{Immune memory clonal selection algorithm.}

Artificial immune system (AIS) is a kind of new intelligent calculation method inspired by biological immune system. De Castro, firstly put forward clonal selection algorithm CLONALG based on principle of clonal selection[14,15]. Immune memory clonal selection algorithm respond the first time invading antigen collection $\mathrm{Ag}$, generate a lot of antibody collection Ab, according to the antigen and antibody affinity to choose the best collection of antibody $n_{a b}$, and memory the antigen and the best antibody. When the antigen occur variation, the system will again be on the immune response.

The main steps of IMCSA are as follows: Encode and initialize antibodies, antigens; Select, clone, mutate antibodies; Calculate affinity, select best antibodies and discard the low-affinity antibodies; Loop calculation, until the antibody which best fits to this antigen is found out, if the number of iterations get the maximum times or affinity get the threshold, the iteration should stopped; Add this antigen and the best antibody in memory; When antigen is mutated, get 
the antibody which is the best match to this mutative antigen, until the iteration meet the termination condition; Select the highest affinity between antibody and antigen in memory; Decode antibodies and antigens, get matching points. Fig. 2 shows the flow chart of IMCSA.

IMCSA describes the antibody and antigen compatibility by affinity function. Feature point matching degree is not only related with the feature points own gray information, also closely connected with the feature points surrounding pixels. The affinity function is as follows.

$$
\operatorname{affinity}(i, j)=\left(0.6-/ M_{A}-M_{B} / / 10\right)\left(\frac{1}{|A(i)-B(j)| / 10+1}\right)+\left(0.4+\mid M_{A}-M_{B} / / 10\right)\left(\frac{\sum_{k}\left(W_{i}\left(x_{k}, y_{k}\right)-u_{i}\right)\left(W_{j}\left(x_{k}, y_{k}\right)-u_{j}\right)}{\sqrt{\sum_{k}\left(W_{i}\left(x_{k}, y_{k}\right)-u_{i}\right)^{2} \sum_{k}\left(W_{j}\left(x_{k}, y_{k}\right)-u_{j}\right)^{2}}}\right)
$$

$M_{A}$ and $M_{B}$ are the average gray values of the image $A$ and $B$ with the limitation $0 \leq\left|M_{A}-M_{B}\right| \leq 50 ; A(i)$ is the i-th feature point gray value of image $A ; B(j)$ is the j-th feature point gray value of image $B ; W_{i}$ and $W_{j}$ are two of the same size window, which bear with feature point as the center; $W_{i}\left(x_{k}, y_{k}\right)$ and $W_{j}\left(x_{k}, y_{k}\right)$ represent respectively the pixel gray value of location $\left(x_{k}, y_{k}\right) ; u_{i}$ and $u_{j}$ is the average of gray values of the window in image $\mathrm{A}$ and image B. Figure 2 is the flow chart of Immune memory clonal selection algorithm.

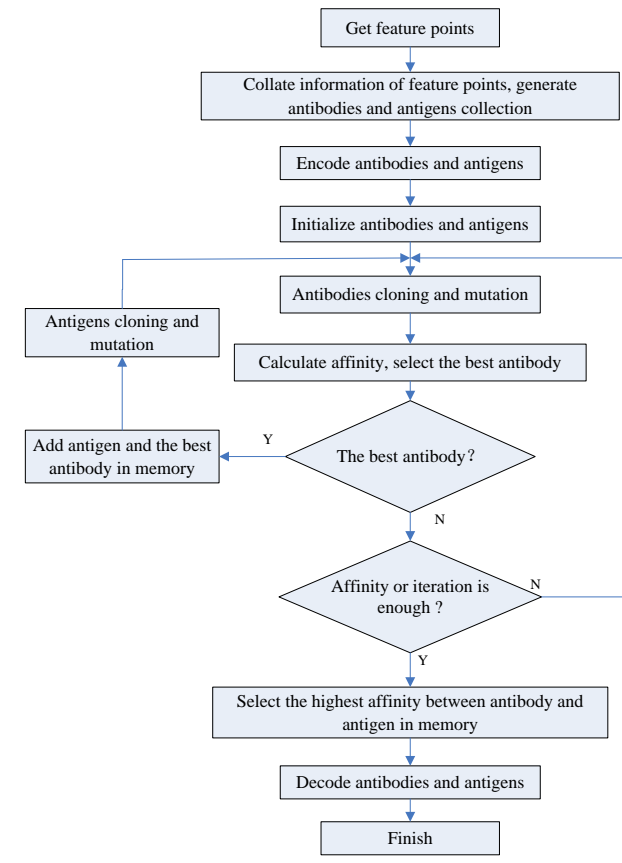

Fig. 2. Immune memory clonal selection algorithm

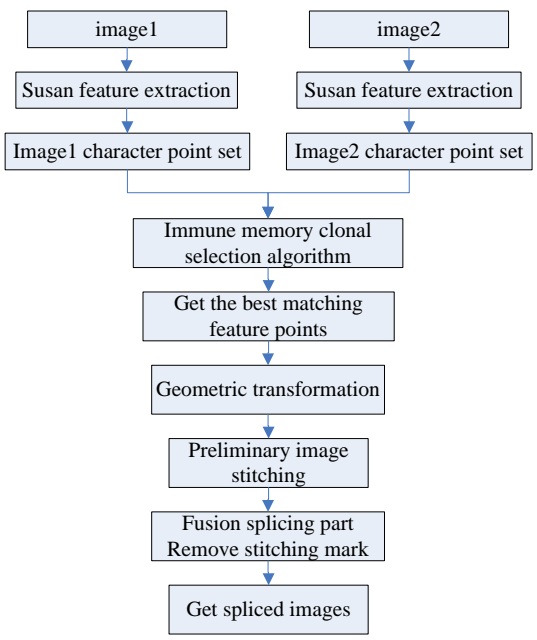

Fig. 3. Image Stitching flow chart

\section{Infrared image stitching process}

In this paper, the infrared image stitching method is based on the characteristics, namely on the premise of at least $3 \sim 5$ best feature points, splice the two images by fusing the common part in a simple manner. Splicing process is shown in figure 3. Firstly, Susan algorithm is applied to extract the feature points. Then combining immune memory clonal selection algorithm, select matching points from feature points, obtain the splice position of the two pictures, determine the stitching coordinate geometry. Finally, fuse the image to get seamless stitching image. Fig. 3 shows the flow chart of Infrared image stitching process.

\section{Simulation and Analysis}

In order to verify the accuracy of matching, this paper split Figure 4 (a) into two parts which is shown in Figure 4 (b) and (c). Because the original image is known, the matching point coordinates in the original image can be obtained by calculation. 


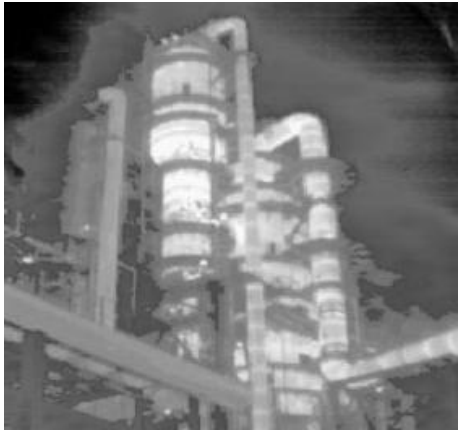

(a) The original graph

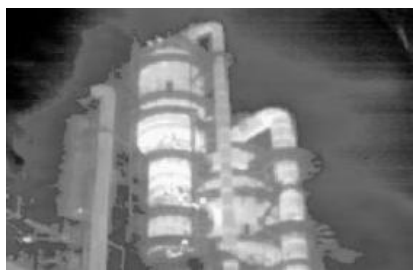

(b) The upper part: image $A$

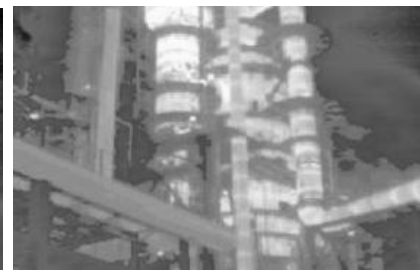

(c) The lower part: image $B$

Fig. 4. The original image for matching search

The feature points set extracted from Image A (Fig.4 (b)) act as antigen set; the feature points set extracted from Image B (Fig. 4 (c)) act as antibody set. Use the given immune memory clonal selection algorithm to search in the collection of antibody and antigen, determine the best matching of antibody and antigen as the matching position. Usually the number of matching points $n \geq 3$, but it Should not be too large. In this paper, we set the number of matching points $\mathrm{n}=5$.

Susan algorithm is adopted to improve the feature point extraction, as shown in Fig. 5 (a) (b). IMCSA is used in the feature point set matching search, the result is shown in Fig. 5 (c), (d).

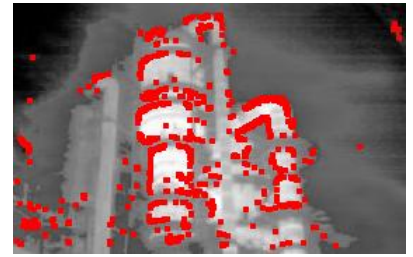

(a) feature points of image $A$

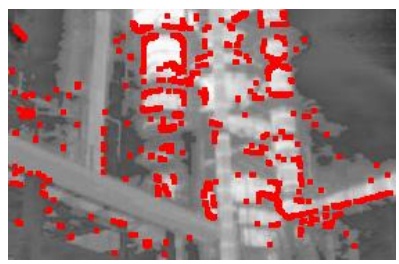

(b) feature points of image $B$

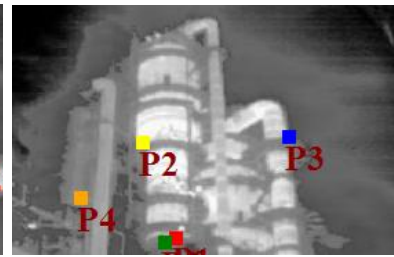

(c) match points of image $A$

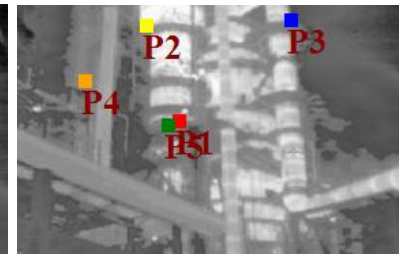

(d) match points of image $B$

Fig. 5. Infrared image matching search simulation results based on IMCSA

By calculating matching points in the original coordinates, results are as follows:

Table 1. The results of infrared image matching search based on IMCSA

\begin{tabular}{|l|l|l|l|l|l|l|c|c|}
\hline & $\begin{array}{l}\text { Matching } \\
\text { point 1 }\end{array}$ & $\begin{array}{l}\text { Matching } \\
\text { point 2 }\end{array}$ & $\begin{array}{l}\text { Matching } \\
\text { point 3 }\end{array}$ & $\begin{array}{l}\text { Matching } \\
\text { point 4 }\end{array}$ & $\begin{array}{l}\text { Matching } \\
\text { point 5 }\end{array}$ & $\begin{array}{l}\text { Original feature } \\
\text { points number }\end{array}$ & $\begin{array}{l}\text { Matching point } \\
\text { number }\end{array}$ & Accuracy \\
\hline Image A & $(118,168)$ & $(94,100)$ & $(200,94)$ & $(49,139)$ & $(110,170)$ & 829 & 5 & \multirow{2}{*}{$100 \%$} \\
\hline Image B & $(118,168)$ & $(94,100)$ & $(200,94)$ & $(49,139)$ & $(110,170)$ & 841 & & 100 \\
\hline
\end{tabular}

As can be seen from the table, in the absence of interference, the matching degree can reach $100 \%$.

When there is a difference between light and shade, the influence of the gray relation between the points around the window center to the affinity is larger and the algorithm is adaptive adjust the weighting coefficient of affinity function.

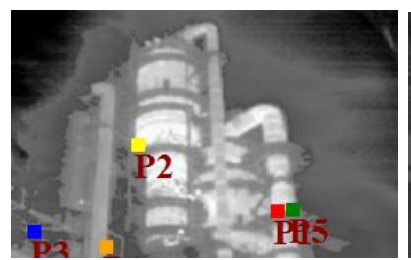

(a) Match points of image $A$

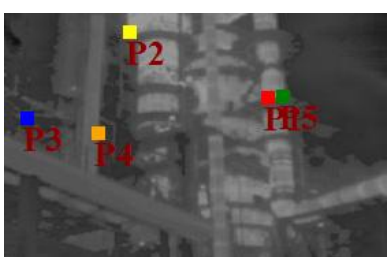

(b) Match points of image $B$

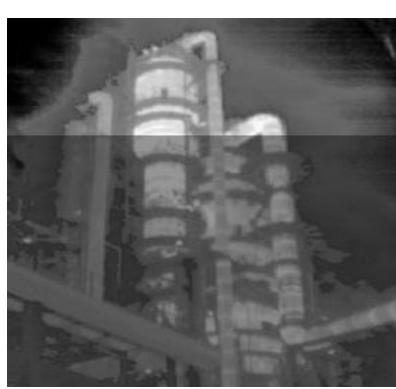

(c) Image matching results

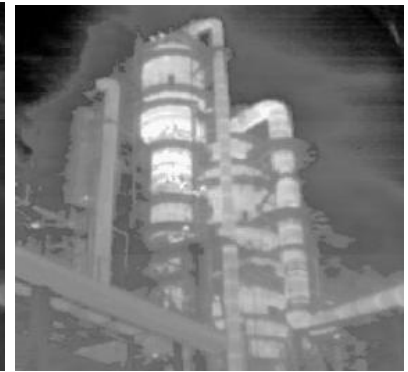

(d) Image fusion results

Fig. 6. Simulation results of infrared image with luminance difference based on IMCSA

Fig. 7.

When the image exist geometric deviation, the algorithm also can achieve good effect of splicing, as shown in 


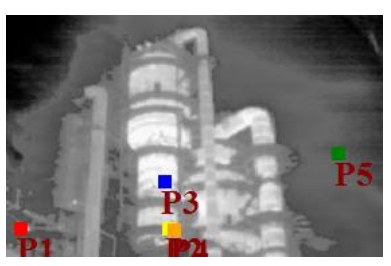

(a) Match points of image $A$

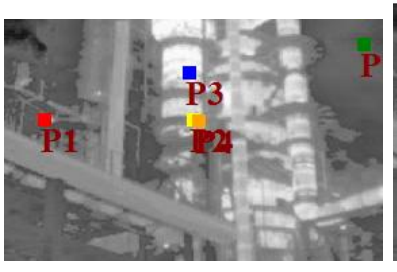

(b) Match points of image $B$

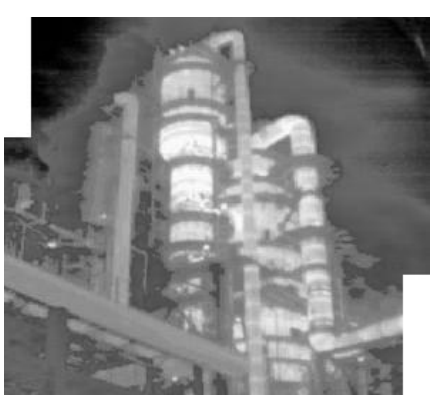

(c) Image Stitching results

Fig. 7. Simulation results of infrared image with geometric deviation based on IMCSA

When the image rotation or scale change, it has a certain influence on affinity function and the simulation results appear a certain error, but by the rest correct matching points, the algorithm can still achieve good stitching. In addition, if continue to increase the number of iterations, we can find out all correct matching feature points. This proves IMCSA has good anti-interference ability.

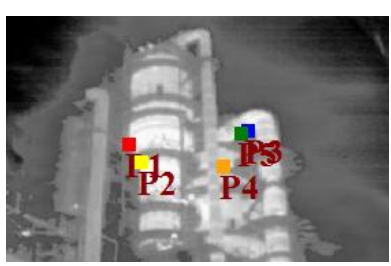

(a) Match points of image $A$

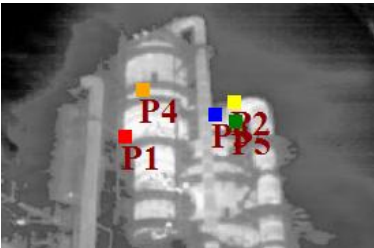

(d) Match points of image $A$

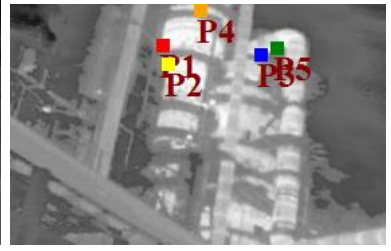

(b) Match points of image $B$

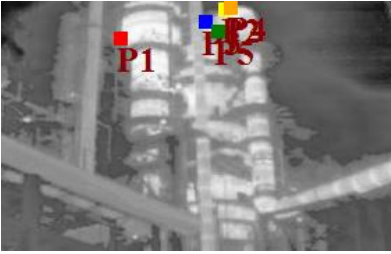

(e) Match points of image $B$

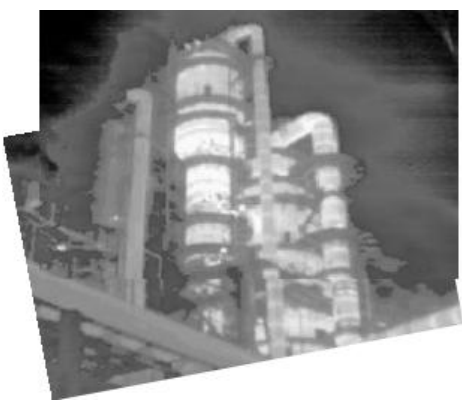

(c) Rotating image Stitching results

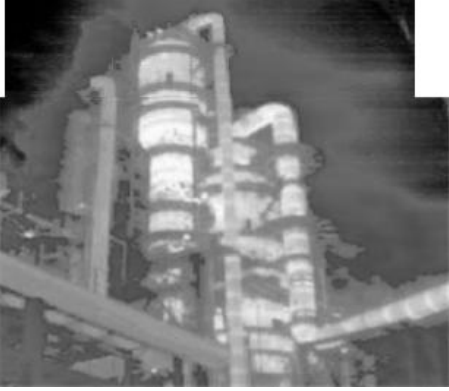

(f) Proportional change image Stitching results

Fig. 8. Simulation results of infrared image with geometrical transformation based on IMCSA

IMCSA is also applied in the other infrared images as follows, and the result shows that the algorithm has good adaptability and robustness.

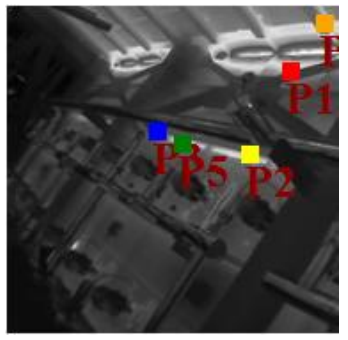

(a) Match points of image $A$

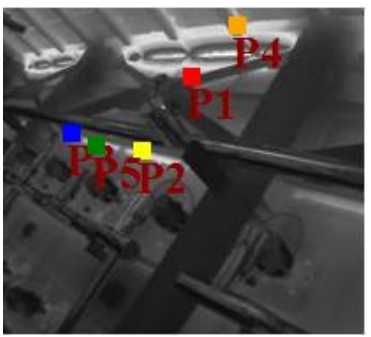

(b) Match points of image $B$

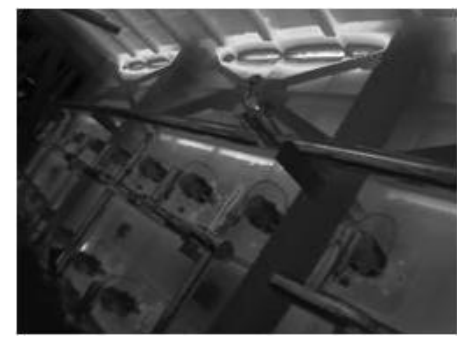

(c) Image Stitching results

Fig. 9. Simulation results of other infrared image based on IMCSA 


\section{Conclusion}

By the simulation results can be seen that the IMCSA can get better stitching matching points, its advantages are mainly as follows:

1) In the absence of interference, the algorithm for stitching matching point accuracy can reach $100 \%$.

2) In the presence of a particular image noise, the inefficiency of feature point extraction, the algorithm result five match points appeared one false matching point. Voting principle was used to most of the matching point for image stitching. This can obtain accurate matching results, and show good anti-jamming capability.

3) The algorithm not only has good stitching effect to the infrared image, for complex background of visible light image also have good stitching effect, shows that the proposed algorithm has good ability to adapt.

4) The algorithm can be adapted to image translation transformation and simple geometric transformation.

5) Due to immune memory exists in the algorithm, good matching result is achieved, at the same time the algorithm has certain preventive to fall into local optimum in the process of search.

\section{REFERENCES}

[1] Zhang Xuewu, Wang Yan, Li Wei, Zhou Yun, "Infrared Image Mosaic Based on Pulse Coupled Neural Network". Applied Mechanics and Materials, vol. 263-266, pp. 2493-2497, 2013.

[2] Jiang Jitong, Song Chao, An Jun, "Study on infrared thermography mosaic algorithm based on the feature point detection and registration". Advanced Materials Research, pp.1138-1142, 2012.

[3] Yang Ling, Cheng Yun, Yue Zhou, and Zhou Taoyun, "Image Mosaic Algorithm Based on Similar Curve" . International Conference on Intelligent System and Knowledge Engineering, pp. 1092-1095, 2008.

[4] Gupta R., Hartley R.I, "Linear Pushbroom Cameras". Pattern Analysis and Machine Intelligence IEEE Transactions on, vol. 19, no. 9, pp. 963-975, 1997.

[5] Li Zhigang, Ji Yubo, Xue Quan, "A Fast Stitching Algorithm for Edge Overlapping Image". Computer Engineering, vol.26, no.5, pp. 37-38, May 2000.

[6] De Castro E., Morandi C., "Registration of Translated and Rotated Images Using Finite Fourier Transforms" . Pattern Analysis and Machine Intelligence IEEE Transactions on, vol. 9, no. 5, pp. 700-703, 1987.

[7] B. Srinivasa Reddy, B. N. Chatterji, "An FFT—based technique for translation, rotation, and scale-invariant image registration". IEEE Transactions on Image Processing, vol. 8, no. 5, pp. 1266-1271, 1996.

[8] Chris Harris, Mike Stephens, "A Combined Corner and Edge Detector". Proceedings of the 4th Alvey Vision Conference, pp. 147-151, 1988.

[9] Smith SM., Brady JM, "SUSAN - A New Approach to Low Level Image Processing". International Journal of Computer Vision, vol. 23, no. 1, pp. 45-78, 1997.

[10] Dong Lin, Fu Dongmei, Yu Xiao, Yang Tao, "The Study on Infrared Image Mosaic Application Using Immune Memory Clonal Selection Algorithm”. 2012 10th World Congress on Intelligent Control and Automation (WCICA), pp. 4831-4836, 2012.

[11] Zhi-guo Qu, Peng Wang, Ying-hui Gao, Ping Wang, Zhen-Kang Shen, "Fast SUSAN edge detector by adapting step-size". Optik, vol. 124, no.8, pp.747-750, 2013.

[12] Chao Gao, HongjunZhu, Yongcai Guo, "Analysis and improvement of SUSAN algorithm". Signal Processing, vol. 90, no.10, pp. 2552-2559, 2012.

[13] Liangyu He, Xingyu Zhou, "An Auto-adaptive Threshold Pre-detection SUSAN Corner Detection Algorithm". 2013 Fifth International Conference on Intelligent Human-Machine Systems and Cybernetics, pp. $511-514$, 2013.

[14] Ma Wenping, Ti Feifei, Li Congling, Jiao Licheng, "Image segmentation based on differential immune clone clustering algorithm". International Journal of Intelligent Computing and Cybernetics, vol. 6, no. 1, pp. 83-102, 2013.

[15] Guan Hongjie, Chen Yuan, Du Peijun, "Study of an improved clonal selection algorithm and its application". International Journal of Digital Content Technology and its Applications, vol. 6, no. 20, pp. 323-332, 2012. 